\title{
Article
}

\section{Transient Current Density in a Pair of Long Parallel Conductors}

\author{
Oldřich Coufal
}

Citation: Coufal, O. Transient Current Density in a Pair of Long Parallel Conductors. Appl. Sci. 2021, 11, 6920. https://doi.org/10.3390/app11156920

Academic Editors: Giovanni Petrone and Fabio La Foresta

Received: 10 June 2021

Accepted: 23 July 2021

Published: 27 July 2021

Publisher's Note: MDPI stays neutral with regard to jurisdictional claims in published maps and institutional affiliations.

Copyright: (C) 2021 by the author. Licensee MDPI, Basel, Switzerland. This article is an open access article distributed under the terms and conditions of the Creative Commons Attribution (CC BY) license (https:// creativecommons.org/licenses/by/ $4.0 /)$.
Department of Electrical Power Engineering, Faculty of Electrical Engineering and Communication, Brno University of Technology, Technicka 12, 61600 Brno, Czech Republic; coufal@vut.cz

\begin{abstract}
Two infinitely long parallel conductors of arbitrary cross section connected to a voltage source form a loop. If the source voltage depends on time, then due to induction there is no constant current density in the loop conductors. It is only recently that a method has been published for accurately calculating current density in a group of long parallel conductors. The method has thus far been applied to the calculation of steady-state current density in a loop connected to a sinusoidal voltage source. In the present article, the method is used for an accurate calculation of transient current using transient current density. The transient current is analysed when connecting and short-circuiting the sources of sinusoidal, constant and sawtooth voltages. For circular cross section conductors, the dependences of maximum current density, maximum current and the time of achieving steady state on the source frequency, the distance of the conductors and their resistivity when connecting the source of sinusoidal voltage are examined.
\end{abstract}

Keywords: transmission and distribution lines; induction; current density; mathematical modelling; ordinary differential equations

\section{Introduction}

The article deals with the calculation of transient current density and transient current in a long loop formed by two long parallel solid conductors of arbitrary cross section and a voltage source. The source voltage is assumed to be dependent on time $t$. Due to induction, the current density in the conductors is a function of $t$ too. It is only recently that a method has been published for accurately calculating current density in a group of long parallel conductors [1]. The method has thus far been applied to the calculation of steady-state current density in a loop connected to a sinusoidal voltage source [2].

The original contributions of the present article are as follows:

- $\quad$ adjustment of the method of current density calculation proposed in [1] to a form suitable for numerical calculation of transient current density;

- $\quad$ presentation of results of the calculation of transient current density and transient current;

- analysis of transient current when connecting on and short-circuiting a voltage source;

- results of the calculation of the dependences of maximum current density, maximum current and the time of achieving steady state on the distance of circular cross section conductors, the frequency of sinusoidal voltage source and the resistivity of conductors.

In the existing methods for the calculation of transient current in a pair of conductors of circular cross section, the current is not determined by transient current density. Either there is a constant current density in the conductor cross section [3] or the loop is replaced by a circuit with lumped parameters. The circuit is a series connection of a resistor with the resistance $R$ and an inductor with the inductance $L$. The quantities $R$ and $L$ are specified prior to the calculation of transient current, which is problematic [2]. Using the proposed method, it is therefore possible to solve problems that have been solved in a steady state (e.g., [4-7]).

In the calculation of current density, a quasi-stationary process is assumed and the displacement current and leakage current between conductors are neglected. The conduc- 
tors do not move. The permeability of the conductors and their surroundings equals the vacuum permeability $\mu_{0}$. In the chosen coordinate system $x y z$, the conductors are parallel to the axis $z$ and their cross sections do not depend on $z$. Figure 1 gives an example of the cross sections of the conductors $\mathscr{A}_{1}$ and $\mathscr{A}_{2}$ in the plane $x y$, the cross sections are denoted by the same symbols as the conductors. The resistivity of the conductors is a function of $x$ and $y, \varrho=\varrho(x, y)$. The voltage source the conductors are connected to produces a potential $V(z, t)$ between the conductors, while the cross section of each conductor is an equipotential area. When calculating the current density, the voltage $U(t)=V\left(z_{1}, t\right)-V\left(z_{2}, t\right) /\left(z_{2}-z_{1}\right)$, where $z_{2}>z_{1}$, is assumed to be specified. The current $I(t)$ in the loop is the flux of current density vector $\mathbf{J}$ through the cross section of one of the loop conductors. $J_{z}(x, y)=J(x, y)$ is the only non-zero component of $\mathbf{J}$.

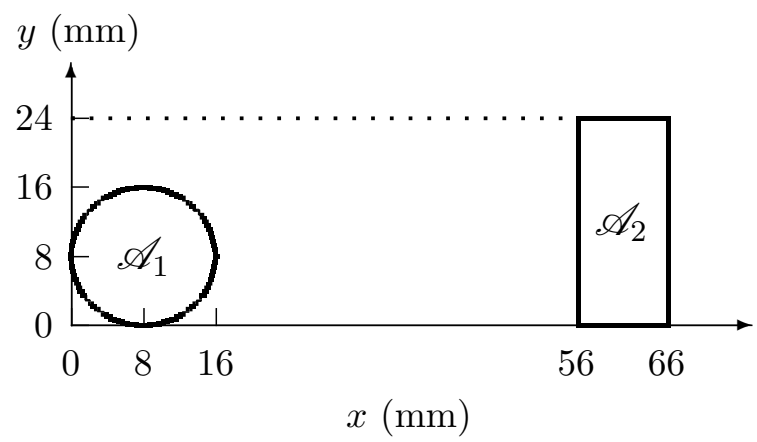

Figure 1. The cross sections of the conductors $\mathscr{A}_{1}$ and $\mathscr{A}_{2}$.

\section{Calculation of Current Density and Current in Conductors}

The essence of the method for calculating current density as published in [1] consists in the conductors being replaced by the partial conductors $A_{i}, \forall i$. The symbol $\forall$ means that the quantity after it takes the values $1,2, \ldots, N$ successively. The cross section of each partial conductor $A_{i}$ is also denoted by the symbol $A_{i}$. Each cross section $\mathscr{A}_{\ell}, \ell=1,2$, can be replaced with arbitrary precision by the union $a\left(\mathscr{A}_{\ell}\right)$ of identical disjunct rectangles that have been formed using the net of parallel lines

$$
x=k_{x} \Delta_{x}, \quad y=k_{y} \Delta_{y},
$$

where $k_{x}, k_{y}$ are integers and $\Delta_{x}$ and $\Delta_{y}$ are the chosen lengths of the rectangle sides. The rectangles of the net, which are subsets in $a\left(\mathscr{A}_{\ell}\right), \ell=1,2$, are the cross sections of the partial conductors $A_{i}, \forall i . A_{i} \subset a\left(\mathscr{A}_{\ell}\right)$ if $\left(X_{i}, Y_{i}\right) \in \mathscr{A}_{\ell}$, where $\left(X_{i}, Y_{i}\right)$ is the centre of the rectangle $A_{i}$. The partial conductors $A_{i}$, which form $a\left(\mathscr{A}_{1}\right)$ and $a\left(\mathscr{A}_{2}\right)$, must be numbered such that the graph with $N$ vertices $\left(X_{i}, Y_{i}\right)$ and $N-1$ edges is a path graph [1,8]. It is assumed that, in the partial conductor $A_{i}, \forall i$, there is a constant resistivity $\varrho_{i}=\varrho\left(X_{i}, Y_{i}\right)$ and that the current density $J_{i}(t)$ does not depend on $x$ and $y$.

According to Coufal [1], the current densities $J_{i}(t), \forall i$, in conductors $\mathscr{A}_{1}$ and $\mathscr{A}_{2}$ are a solution to the system of $N-1$ ordinary differential equations

$$
\varrho_{i} J_{i}(t)-\varrho_{i+1} J_{i+1}(t)+\frac{\mu_{0}}{4 \pi} \sum_{k=1}^{N} \phi_{i k} \dot{J}_{k}(t)=\delta_{i} U(t), \quad i=1,2, \ldots, N-1,
$$

and one algebraic equation

$$
\sum_{k=1}^{N} J_{k}(t)=0
$$

The differentiation of a function with respect to time $t$ is denoted by a dot over the symbol of the function. The coefficients $\phi_{i k}$ do not depend on $t$, and their calculation is described in the Appendix of [9]. $J_{k}(t)\left(z_{2}-z_{1}\right) \mu_{0} \phi_{i k} /(4 \pi)$ is the contribution of the $k$ th partial conductor to the magnetic flux through the segment (between the planes $z=z_{1}$ and 
$z=z_{2}$ ) of the loop formed by partial conductors $A_{i}$ and $A_{i+1}$. The value of the coefficient $\delta_{i}$ on the right-hand side of Equation (1) is given by the relation

$$
\delta_{i}=\left\{\begin{aligned}
1 & \text { if }\left(A_{i} \subset a\left(\mathscr{A}_{1}\right)\right) \wedge\left(A_{i+1} \subset a\left(\mathscr{A}_{2}\right)\right), \\
-1 & \text { if }\left(A_{i} \subset a\left(\mathscr{A}_{2}\right)\right) \wedge\left(A_{i+1} \subset a\left(\mathscr{A}_{1}\right)\right), \\
0 & \text { otherwise. }
\end{aligned}\right.
$$

Prior to the numerical solution to the system formed by Equations (1) and (2), Equation (2) is substituted by the equation

$$
\sum_{k=1}^{N} \dot{J}_{k}(t)=0
$$

The system of Equations (1) and (4) can after rearrangement be replaced by a single equation

$$
\phi \dot{j}(t)=10^{7} \mathrm{D}+10^{7} U(t) \delta,
$$

where the matrix $\phi$ is of the type $(N, N)$, on whose first $N-1$ rows are the fluxes $\phi_{i k}, \forall k, i=1,2, \ldots, N-1$, while on the $N$ th row are ones (coefficients on the left-hand side of Equation (4)); $10^{7}=4 \pi / \mu_{0}$; the matrices $\mathrm{J}$, D and $\delta$ are of the type $(N, 1) . \dot{j}=\left[\dot{j}_{i}(t)\right]$; $\mathrm{D}=\left[D_{i}\right], D_{i}=\varrho_{i+1} J_{i+1}-\varrho_{i} J_{i}$ for $i=1,2, \ldots, N-1, D_{N}=0 . \delta=\left[\delta_{i}\right], \delta_{i}$ is defined by relation (3) for $i=1,2, \ldots, N-1, \delta_{N}=0$. Multiplying Equation (5) by the inverse matrix $\phi^{-1}$ from the left yields the equation

$$
\mathrm{j}=10^{7} \mathrm{M}_{J}+10^{7} U(t) \mathrm{M}, \quad \text { where } \mathrm{M}_{J}=\phi^{-1} \mathrm{D}, \mathrm{M}=\phi^{-1} \delta .
$$

The matrices $M_{J}$ and $M$ are of the type $(N, 1)$. The rows of the matrix $M_{J}$ are linear combinations of the current densities $J_{i}(t)$. The elements of matrix $\mathrm{M}$ are numbers. Equation (6) is a linear inhomogeneous system of ordinary differential equations with constant coefficients. According to Rektorys and Vitasek [10], there is just one solution $\mathrm{J}=\left[J_{i}(t)\right]$ to Equation (6) in the interval $t \in[0, \infty)$ that satisfies the initial conditions

$$
\mathrm{J}(0)=\mathrm{J}_{0}, \quad \mathrm{~J}_{0}=\left[J_{i}(0)\right] .
$$

The results given below for the solution to Equation (6) were obtained using the Runge-Kutta method [10-12]. Equation (6) can be written in the form

$$
\left[\dot{J}_{i}(t)\right]=\left[F_{i}\left(t, J_{k}(t), \forall k\right)\right] .
$$

If $J_{i}(t), \forall i$, is the current density in the $i$ th partial conductor at time $t$, then

$$
J_{i}(t+h)=J_{i}(t)+\frac{1}{6}\left(k_{1 i}+2 k_{2 i}+2 k_{3 i}+k_{4 i}\right)
$$

is the current density in the $i$ th partial conductor at time $t+h$, where $h$ is the integration step,

$$
\begin{aligned}
& k_{1 i}=h F_{i}\left(t, J_{k}(t), \forall k\right), \\
& k_{2 i}=h F_{i}\left(t+h / 2, J_{k}(t)+k_{1 k} / 2, \forall k\right), \\
& k_{3 i}=h F_{i}\left(t+h / 2, J_{k}(t)+k_{2 k} / 2, \forall k\right), \\
& k_{4 i}=h F_{i}\left(t+h, J_{k}(t)+k_{3 k}, \forall k\right) .
\end{aligned}
$$

All the values of transient current $I(t)$ given below were calculated using the transient current density in the conductor $\mathscr{A}_{1}$

$$
I(t)=\Delta_{x} \Delta_{y} \sum_{i} J_{i}(t), \text { where } A_{i} \subset a\left(\mathscr{A}_{1}\right) .
$$

The current in the conductor $\mathscr{A}_{2}$ is $-I(t)$. 


\section{Results and Discussion}

\subsection{Example 1}

The cross sections of the conductors forming the long loop are illustrated in Figure 1. The temperature of the conductors is $300 \mathrm{~K}$, both conductors are of copper and their resistivity $\varrho=1.725 \times 10^{-8} \Omega \cdot \mathrm{m}[13]$. At time $t=0$, the conductors are connected to the sinusoidal voltage source $U(t)=\hat{U} \sin (\omega t+\alpha), \hat{U}=1 \mathrm{~V} \cdot \mathrm{m}^{-1}$, the frequency $f=\omega /(2 \pi)$ and the period $T=1 / f$.

This example is for $f=1 \mathrm{kHz}$, the same as in [2], Variant 4; however, in Figure 6 in [2], the results of the calculation of current density in conductors are only given for the steady state, i.e., for $t \rightarrow \infty$. Figure 2 illustrates the dependences of current density on $x$ and $t$ for a constant $y$ in the conductors $\mathscr{A}_{1}$ and $\mathscr{A}_{2}$.

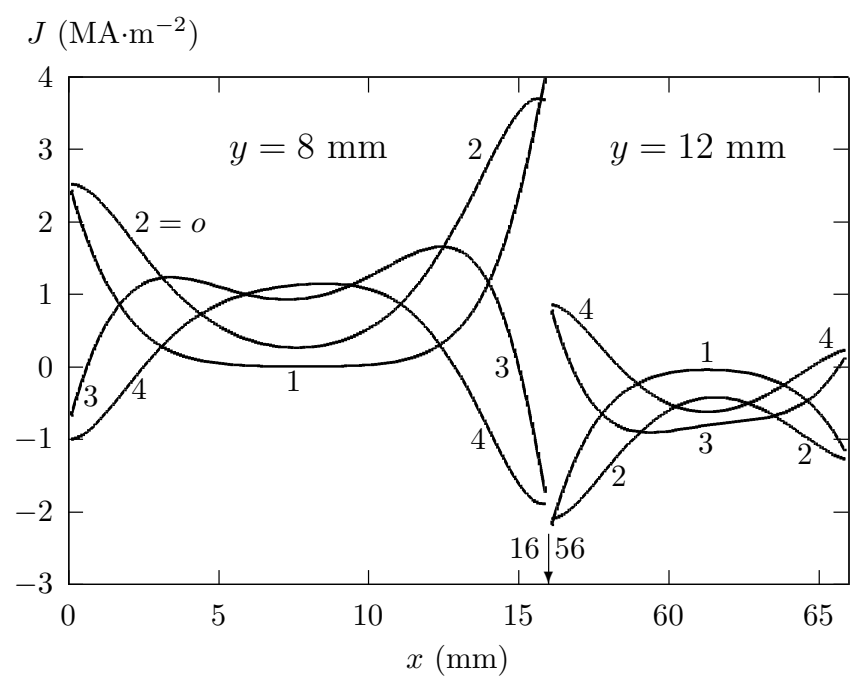

Figure 2. Example 1, $\alpha=0, f=1 \mathrm{kHz}$ : The dependences of current density on $x$ and $t$ for a constant $y$ in the conductors $\mathscr{A}_{1}$ and $\mathscr{A}_{2}$. The curve marked by the character $o$ represents the current density at time $t=o \times T / 4 ; J=0$ for $t=0$. The axis $x$ at the point marked by the arrow ends in the value $x=16 \mathrm{~mm}$ and continues with the value $x=56 \mathrm{~mm}$. Between these values is the gap between the conductors, and the current density is zero.

The calculation of transient current density provides a large amount of data that can be displayed in a similar way to Figure 2. For example, the maximum value of $\left|J_{i}\right|$ is equal to $6.095 \mathrm{MA} \cdot \mathrm{m}^{-2}$ in the partial conductor that is down on the left in the conductor $\mathscr{A}_{2}$, in the interval $t \in[0, T]$ at time $t=3.3 \times 10^{-4} \mathrm{~s}$, for $\alpha=0, f=1 \mathrm{kHz}$.

The current densities $J_{i}(t), \forall i$, are non-periodic oscillating functions that with increasing $t$ converge to periodic functions. For $t \rightarrow \infty$, it can be assumed that for the sinusoidal voltage source $\hat{U} \sin (\omega t+\alpha)$ and the cosinusoidal voltage source $\hat{U} \cos (\omega t+\alpha)$ the current density in the $i$ th partial conductor is

$$
J_{i}(t)=\hat{J}_{i} \sin \left(\omega t+\varepsilon_{i}\right), \forall i
$$

and

$$
J_{i}(t)=\hat{J}_{i} \cos \left(\omega t+\varepsilon_{i}\right), \forall i
$$

respectively. Substituting the assumed solutions to (8) and (9) into (1) and (4) yields the first and second equation systems, respectively. The two systems form a system of $2 \mathrm{~N}$ equations for $N$ unknown amplitudes $\hat{J}_{i}$ and $N$ unknown initial phases $\varepsilon_{i}$. Probably the simplest way of finding a solution to the two systems is to multiply the first system by the imaginary unit $j$ and add the second system of equations. This after adjustment yields a system of $N$ equations for the complex current densities $\hat{J}_{i} \exp \left(\mathrm{j} \varepsilon_{i}\right) \exp (\mathrm{j} \omega t), \forall i$. Solving this system gives current density phasors in the partial conductors, as stated in [1].

With increasing $t$, the transient current converges to the steady sinusoidal current 


$$
I_{\sin }(t)=\hat{I} \sin (\omega t+\beta) .
$$

Figure 3 illustrates the current $I(t)$ in three time intervals.

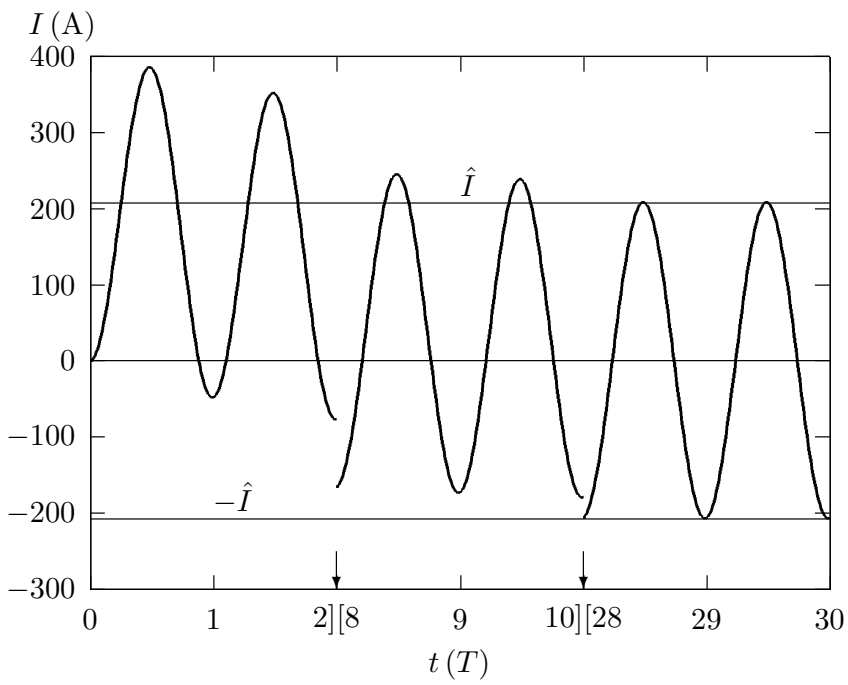

Figure 3. Example 1, $\alpha=0, f=1 \mathrm{kHz}$ : The dependence of current on $t$ in three time intervals: $[0,2 T]$, $[8 T, 10 T]$ and $[28 T, 30 T]$.

Theoretically speaking, the steady state is achieved after an infinitely long time. In practice, the steady state is reached at time $t \geq t_{\sin }$, where $t_{\sin }$ is determined using the chosen value of the deviation $\Delta_{I}$. $t_{\sin }$ is such a least time that for $t \geq t_{\sin }$ it holds

$$
|1-| I_{\operatorname{lm}}(t)|/ \hat{I}| \leq \Delta_{I},
$$

where $I_{\operatorname{lm}}(t)$ is the value of the current at the local maximum or minimum. In Example $1(\alpha=0, f=1 \mathrm{kHz})$, it holds $t_{\sin }=29.49 \mathrm{~T}$ for $\Delta_{I}=0.0032$. For $\Delta_{I}=0.003$ in Example 1 , it holds $t_{\text {sin }} \doteq 0.03-0.04 \mathrm{~s}$ for $f \in\left[50,10^{6}\right] \mathrm{Hz}$. The time $t_{\text {sin }}$ depends substantially on the initial phase $\alpha$ of the voltage source at time $t=0$. In Figure 4 , the function $I(t)$ is illustrated for several values $\alpha$ in Example 1 for $f=1 \mathrm{MHz}$. It can be seen in Figure 4 that the steady state is achieved at the instant $t=0$ for $\alpha_{\sin }=90^{\circ}$. The value $\alpha_{\sin }$ depends on $f$. For example, for $f=50 \mathrm{~Hz}, \alpha_{\sin }=60^{\circ}$.

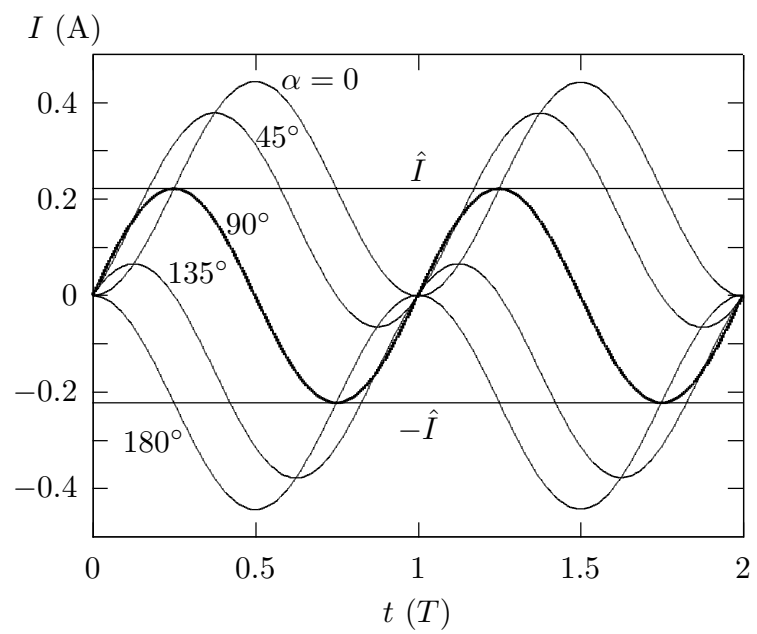

Figure 4. Example 1, $f=1 \mathrm{MHz}$ : The dependence of the current $I(t)$ on the initial voltage phase $\alpha$ for $t \in[0,2 T]$. 


\subsection{Example 2}

The conductors are the same as in Example 1, but, in contrast to Example 1, they are connected to the constant voltage source $U(t)=\hat{U}, \hat{U}=1 \mathrm{~V} \cdot \mathrm{m}^{-1}$ at time $t=0$. The voltage source is being short-circuited at time $t_{\text {sho }}$.

Figure 5 illustrates the function $I(t)$ when the voltage source is being connected and later short-circuited. The graph of the function $I(t)$ has two parts. The first is for $t \in\left[0, t_{\text {sho }}\right]$ increasing while the second part is decreasing in the interval $t \in\left(t_{\text {sho }}, \infty\right)$.

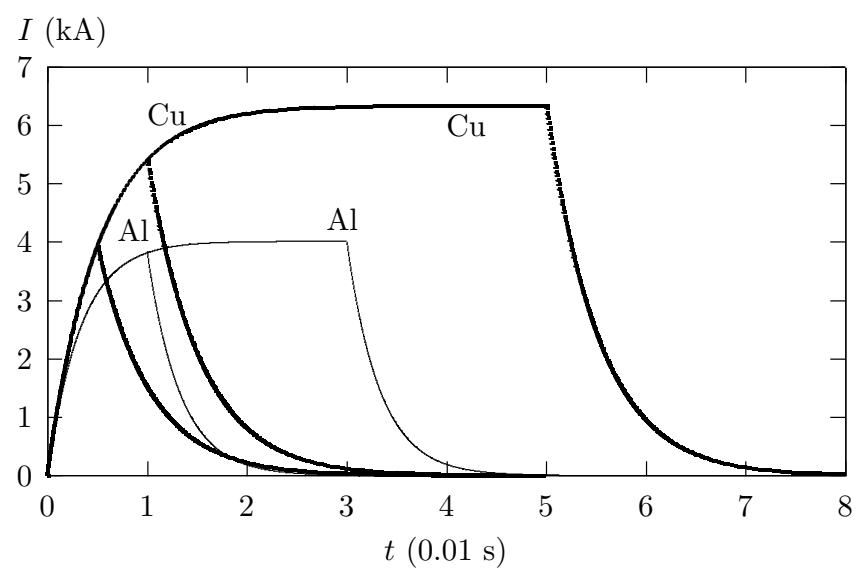

Figure 5. Example 2: The dependence of transient current on time when the source of constant current is connected at time $t=0$ and is later short-circuited at time $t_{\text {sho }}=0.005,0.01,0.05 \mathrm{~s}$. The curves (solid lines) denoted by $\mathrm{Cu}$ correspond to copper conductors. The curves (thin lines) denoted by $\mathrm{Al}$ correspond to aluminium conductors at a temperature of $300 \mathrm{~K}, \varrho=2.733 \times 10^{-8} \Omega \cdot \mathrm{m}[13]$; $t_{\text {sho }}=0.01,0.03 \mathrm{~s}$.

If Figure 5 is redrawn in semilogarithmic coordinates, then the second part of the graph of the function $I(t)$ is a straight line. This means that $\ln I(t)$ is a linear function of $t$ and that the slope $|\Delta \ln I(t)| / \Delta t$ is constant. It follows from the results in Figure 5 that with increasing conductor resistivity the time $t_{\sin }$ decreases and the slope of the drop in current increases after the short-circuiting of the voltage source.

It would undoubtedly be interesting to investigate the disconnection of the source. However, solving such a problem is beyond the scope of the article. Source disconnection is realised using a switch, in which an electric arc is excited between the switch contacts. To calculate transient current density and transient current when disconnecting the source, it is necessary to replace the source by a physical and mathematical model of the concrete switch.

\subsection{Example 3}

The cross section of the two conductors is a circle with the radius $r=8 \mathrm{~mm}$. The cross sections $\mathscr{A}_{1}$ and $\mathscr{A}_{2}$ have their centres at points $(0,0)$ and $(d, 0)$, respectively, $d \geq 2 r$. Both conductors are of copper and they are connected to a sinusoidal voltage source at time $t=0$, the same as in Example 1.

The solution to Example 3 allows extending and complementing the results of solving Example 1. With the sinusoidal source connected, the main effect of the transient state is an increase in the maximum value of the current density

$$
J_{\max }=\max _{\forall i, t \in[0, T)}\left|J_{i}(t)\right|
$$

and in the maximum value of the current

$$
I_{\max }=\max _{t \in[0, T)}|I(t)|
$$


with respect to the steady state. The maximum values $I_{\max }$ and $J_{\max }$ are obtained during the first period $T$. The amplitude of the steady current density attains the maximum

$$
\hat{J}_{\max }=\max _{\forall i} \hat{J}_{i}
$$

at the point $(r, 0)$. The point at which the transient current density attains the value $J_{\max }$ is on the surface of the conductor $\mathscr{A}_{1}$ (and at the symmetrical point on the surface of the conductor $\mathscr{A}_{2}$ ) and its position depends on $d$ and $f$. The instant at which $J_{\max }$ is attained differs from the instant at which $I_{\max }$ is attained (see Figure 6).

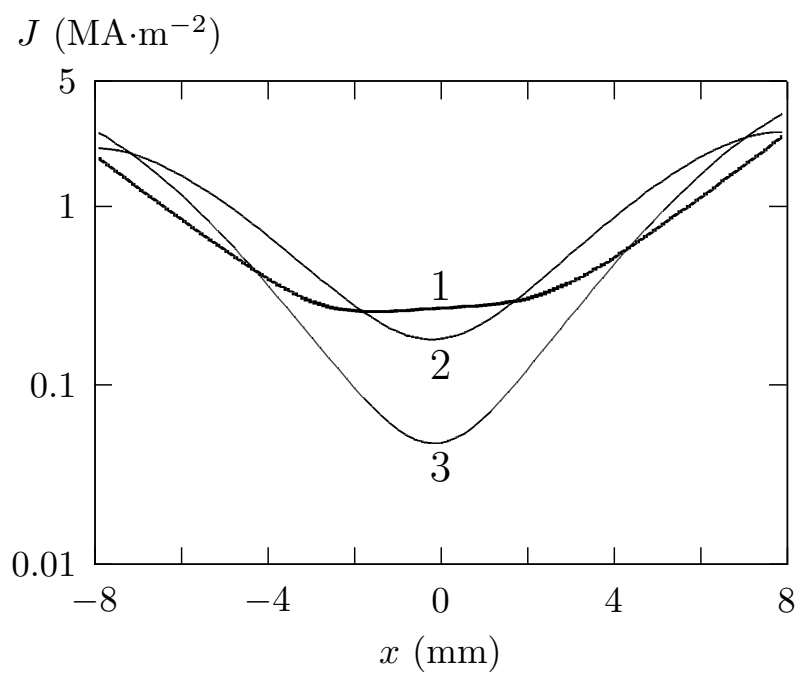

Figure 6. Example 3, $d=100 \mathrm{~mm}, f=1 \mathrm{kHz}, \alpha=0$ : The dependence of current density on $x$ for $y=0$ in the conductor $\mathscr{A}_{1}$. (1) The amplitude of current density in steady state; (2) $t=0.49 \mathrm{~ms}$, the value of the current $I$ is maximum; (3) $t=0.37 \mathrm{~ms}$, maximum current density.

Table 1 gives the values $J_{\max } / \hat{J}_{\max }$ and $I_{\max } / \hat{I}$ in dependences on $f, d$ and $\varrho$. The resistivity of Al conductors is $\varrho=2.733 \times 10^{-8} \Omega \cdot \mathrm{m}$ [13]. Alloy conductors are of an alloy of 75 mass $\% \mathrm{Cu}+25$ mass $\% \mathrm{Al}, \varrho=1.76 \times 10^{-7} \Omega \cdot \mathrm{m}[13]$.

Table 1. Example 3, $\alpha=0$ : The dependences of $M_{J}=J_{\max } / \hat{J}_{\max }$ and $M_{I}=I_{\max } / \hat{I}$ on $f, d$ and $\varrho$.

\begin{tabular}{cccccccc}
\hline$f(\mathbf{H z})$ & $d=$ & $\begin{array}{c}\mathbf{1 6} \mathbf{~ m m} \\
\mathbf{C u}\end{array}$ & $\begin{array}{c}\mathbf{3 2} \mathbf{~ m m} \\
\mathbf{C u}\end{array}$ & $\begin{array}{c}\mathbf{0 . 1} \mathbf{~ m} \\
\mathbf{C u}\end{array}$ & $\begin{array}{c}\mathbf{1} \mathbf{~ m} \\
\mathbf{C u}\end{array}$ & $\begin{array}{c}\mathbf{1} \mathbf{~ m} \\
\mathbf{A l}\end{array}$ & $\begin{array}{c}\mathbf{1} \mathbf{~ m} \\
\text { Alloy }\end{array}$ \\
\hline 50 & $M_{J}$ & 1.001 & 1.082 & 1.232 & 1.441 & 1.291 & 1.002 \\
$10^{3}$ & $M_{J}$ & 1.005 & 1.250 & 1.358 & 1.421 & 1.439 & 1.599 \\
$10^{5}$ & $M_{J}$ & 1.666 & 1.802 & 2.057 & 2.236 & 1.499 & 1.425 \\
\hline 50 & $M_{I}$ & 1.033 & 1.106 & 1.248 & 1.453 & 1.294 & 1.002 \\
$10^{3}$ & $M_{I}$ & 1.537 & 1.801 & 1.890 & 1.939 & 1.920 & 1.659 \\
$10^{5}$ & $M_{I}$ & 1.818 & 1.979 & 1.990 & 1.995 & 1.993 & 1.983 \\
\hline
\end{tabular}

The transient current $I(t)$ can be considered steady for $t \geq t_{\sin }$. The instant $t_{\sin }$ is determined by Formula (10) and depends on the chosen value $\Delta_{I}$, the distance $d$ of the axes of conductor cross sections, the voltage source frequency $f=1 / T$ and the conductor resistivity. Table 2 gives the values of $t_{\sin }$ for several values of $f, \Delta_{I}$ and $d$. The conductors in Example 3 are of copper and the respective data are given in Table 2, with the exception of the last column, where the data given hold for aluminium conductors $\left(\varrho=2.733 \times 10^{-8} \Omega \cdot \mathrm{m}[13]\right)$. With increasing conductor resistivity, the time $t_{\sin }$ decreases. This statement is supported by comparing the values in the penultimate and last columns of Table 2. 
Table 2. Example 3, $\alpha=0$ : The dependences of time $t_{\text {sin }}$ (expressed by the number of whole periods $T$ and rounded up) on $f, \Delta_{I}$ and $d$.

\begin{tabular}{ccccccc}
\hline$f(\mathbf{H z})$ & $\boldsymbol{\Delta}_{\boldsymbol{I}}(-)$ & $\mathbf{1 6} \mathbf{~} \mathbf{m}$ & $\mathbf{3 2} \mathbf{~} \mathbf{m}$ & $\mathbf{0 . 1} \mathbf{~}$ & $\mathbf{1 ~ \mathbf { ~ }}$ & $\mathbf{1} \mathbf{~ m , ~ A l ~}$ \\
\hline 50 & 0.02 & 2 & 2 & 2 & 3 & 2 \\
$10^{3}$ & 0.02 & 9 & 16 & 26 & 47 & 30 \\
$10^{5}$ & 0.02 & 525 & 1425 & 2478 & 4574 & 2887 \\
\hline 50 & 0.01 & 2 & 2 & 3 & 4 & 3 \\
$10^{3}$ & 0.01 & 11 & 18 & 31 & 55 & 35 \\
$10^{5}$ & 0.01 & 695 & 1695 & 3000 & 5394 & 3405 \\
\hline 50 & 0.005 & 2 & 2 & 3 & 4 & 3 \\
$10^{3}$ & 0.005 & 13 & 21 & 35 & 64 & 40 \\
$10^{5}$ & 0.005 & 864 & 1965 & 3380 & 6215 & 3923 \\
\hline
\end{tabular}

\subsection{Example 4}

The conductors are the same as in Example 3. At time $t=0$, they are connected to a source of sawtooth voltage (see Figure 7). The voltage is periodic, in the first period

$$
U(t)=\hat{U}(1-2 t / T), \quad \hat{U}=1 \mathrm{~V} \cdot \mathrm{m}^{-1}, t \in[0, T) .
$$

The solution to Example 4, obtained by solving Equation (6), is given in Figure 7. In the interval $t \in[0,2 T)$, the current $I(t)$ is transient, and for $t \geq 2 T$ the current can be considered steady.

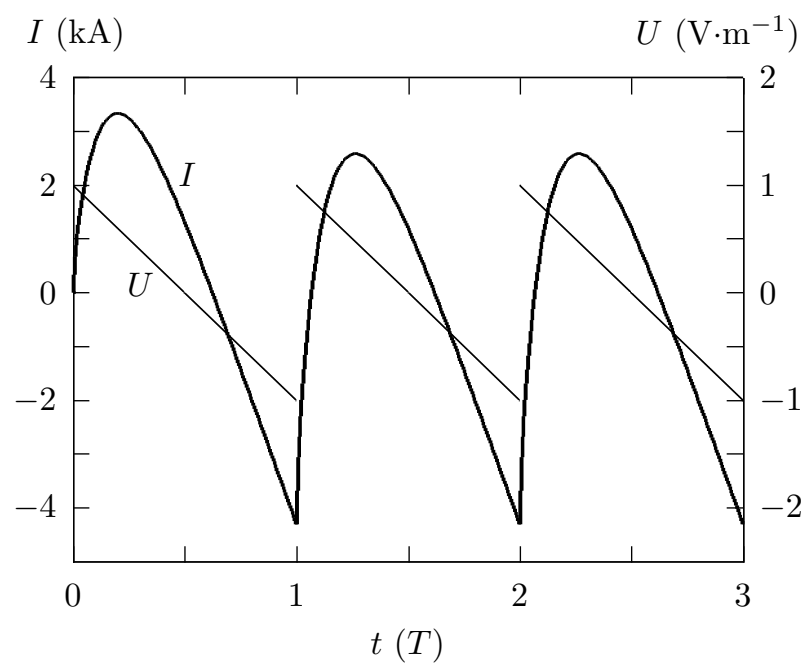

Figure 7. Example $4, d=16 \mathrm{~mm}, f=60 \mathrm{~Hz}, \hat{U}=1 \mathrm{~V} \cdot \mathrm{m}^{-1}$ : The dependences of the current $I$ in the conductor $\mathscr{A}_{1}$ and the voltage $U$ on time $t$.

In the theory of circuits with lumped parameters, the non-sinusoidal voltage (thus also (11)) is in the calculation of steady current $I(t)$ approximated by the first $n$ terms of the Fourier series [14]. For a source voltage that is equal to the $k$ th harmonic, $k=1,2, \ldots, n$, the current $I_{k}(t)$ is then calculated. The principle of superposition is used to obtain the current

$$
I(t)=\sum_{k=1}^{n} I_{k}(t)
$$

An advantage of such a calculation is that it allows the use of phasors. The Fourier series can also be used in the calculation of steady current density, and thus also the steady current, in two parallel long conductors connected to a source of non-sinusoidal voltage via the method published in [1]. When assessing the suitability of such a procedure, it is 
necessary to take into account that the Fourier expansion is an infinite series while the calculation can only be performed with a finite number $n$ of series terms. The finite number of terms only approximates the given voltage course, which also manifests itself in the resultant current. Instead of $n$ repetitions of the calculation with individual harmonics, a single calculation can be done of transient current density and current with the given voltage. The transient current at time $t \geq t_{\text {sin }}$ can be considered steady. Figure 8 gives the sawtooth voltage in Example 4 and its approximation by $n$ terms of the Fourier series [14]

$$
U(t)=\frac{2 \hat{U}}{\pi} \sum_{k=1}^{\infty} \frac{\sin (k \omega t)}{k}
$$

and the transient current calculated with the source voltage approximated by $n$ terms of the expansion (12) (an accurate transient current is in Figure 7).

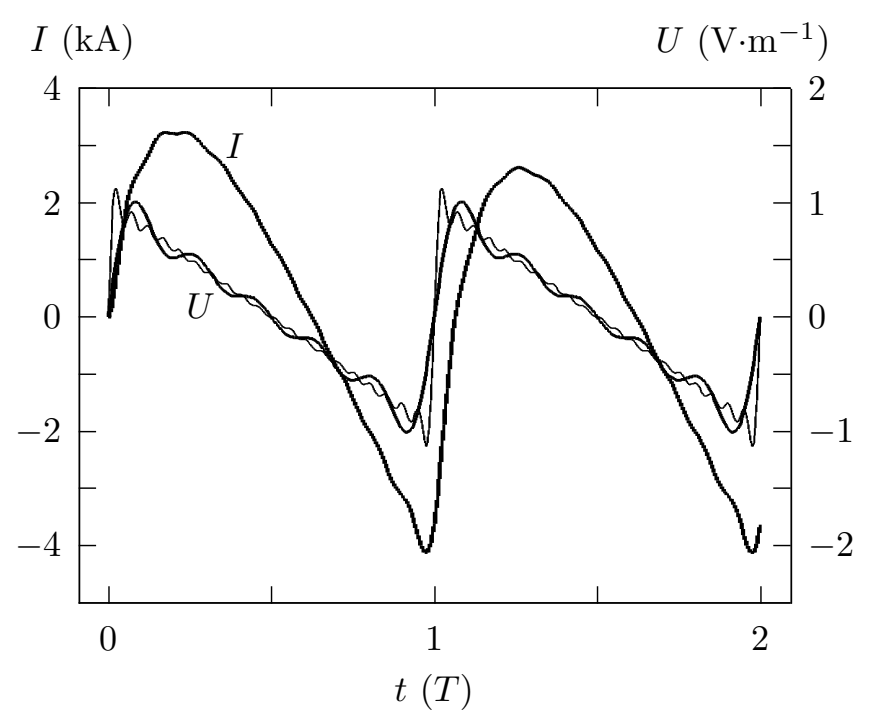

Figure 8. Example $4, d=16 \mathrm{~mm}, f=60 \mathrm{~Hz}, \hat{U}=1 \mathrm{~V} \cdot \mathrm{m}^{-1}$ : Approximation of the voltage $U(t)$ by $n$ terms of the series (12) for $n=5$ (solid line) and $n=20$ (thin line). The current $I$ in the conductor $\mathscr{A}_{1}$ when approximating the voltage by ten terms of the series (12).

\subsection{Discussion}

J. C. Maxwell proposed a method for the calculation of current density in a solitary conductor [15]. Hundreds of works have since been published (more recently, e.g., [1,16-24]) that deal with the calculation of current density in one or two conductors, and exceptionally in more conductors. The calculation of current density in one conductor is accurate, but it can only be applied to the solitary conductor connected to the ideal current source, which, however, cannot be implemented [1]. The published methods, with the exception of those in $[1,3,24]$, are approximate, with the current density error unknown. The self-inductance of two conductors cannot be calculated without accurate current density [2] and thus approximate formulae continue to be used for the calculation of self-inductance of two coaxial conductors and two circular cross section conductors [2]. According to these formulae, inductance does not depend on conductor resistivity and voltage source frequency, in contrast to the inductance calculated using accurate current density.

The subject of the present article is transient current in two solid long parallel conductors (conductors in the following) connected to the voltage source. A definite contribution of the article is that current is determined using transient current density. In the article, a numerical method is proposed and used for solving a system of differential equations for the calculation of transient current density. The present method of calculating transient current, not only in the conductors considered in the present article, consists in calculating transient current in the circuit $\mathscr{C}$ with lumped elements, which is a series connection of 
a resistor (with resistance $R$ ), an inductor (with inductance $L$ ) and a voltage source (with voltage $U$ ). For the circuit $\mathscr{C}$, Kirchhoff's voltage law holds

$$
L \dot{I}(t)+R I(t)=U(t) .
$$

Dividing both sides in (13) by $L$ gives the equation

$$
\dot{I}(t)+\frac{R}{L} I(t)=\frac{U(t)}{L},
$$

which is an ordinary linear inhomogeneous differential equation. According to Rektorys and Vitasek [10], there exists just one solution $I(t)$ to Equation (14) in the interval $0 \leq t<\infty$ that satisfies the given initial condition $I(0)=I_{0}$

$$
I(t)=\exp \left(-\frac{R}{L} t\right)\left[C+\int \frac{U(t)}{L} \exp \left(\frac{R}{L} t\right) \mathrm{d} t\right],
$$

where the constant $C$ is determined by the initial condition.

For the source of sinusoidal voltage $U(t)=\hat{U} \sin (\omega t+\alpha)$, the transient current is

$$
\begin{gathered}
I(t)=K\left[\frac{R}{L} \sin (\omega t+\alpha)-\omega \cos (\omega t+\alpha)\right]+C \exp \left(-\frac{R}{L} t\right), \\
K=\hat{U}\left[\frac{R^{2}}{L}+L \omega^{2}\right]^{-1} .
\end{gathered}
$$

For the initial condition $I(0)=I_{0}$, it holds that

$$
C=I_{0}-K\left(\frac{R}{L} \sin \alpha-\omega \cos \alpha\right) .
$$

Let us consider the $\mathrm{Al}$ conductors $\mathscr{A}_{1}$ and $\mathscr{A}_{2}$ at a temperature of $300 \mathrm{~K}$, $\varrho=2.733 \times 10^{-8} \Omega \cdot \mathrm{m}[13]$. The cross section of the two conductors is a circle the radius $r=8 \mathrm{~mm}$. The cross sections $\mathscr{A}_{1}$ and $\mathscr{A}_{2}$ have their centres at points $(0,0)$ and $(d, 0)$, respectively, $d=2 r$. At the instant $t=0$, the conductors are connected to the source of sinusoidal voltage $U(t)=\hat{U} \sin \omega t, \hat{U}=1 \mathrm{~V} \cdot \mathrm{m}^{-1}, f=10^{5} \mathrm{~Hz}$. The inductance $L$ of the conductor pair can be established using the formula $[2,15]$

$$
L_{\mathrm{lit}}=\frac{\mu_{0}}{\pi}\left[\frac{1}{4}+\ln \frac{d-r}{r}\right] .
$$

According to this formula, $L_{\mathrm{lit}}=10^{-7} \mathrm{H} \cdot \mathrm{m}^{-1}$. Figure 9 illustrates the voltage $U(t)$, the transient current $I_{(16)}(t)$ calculated using formula (16) and the transient current $I(t)$ calculated by the method proposed in the present article for $t \in[0,4 T], I(0)=0$. As mentioned above, the value of $L_{\mathrm{lit}}$ is not accurate. If the accurate value $L=8.404 \times 10^{-8} \mathrm{H} \cdot \mathrm{m}^{-1}$, determined as in [2], were used in (16), the difference between the transient currents would be even greater than in Figure 9. 


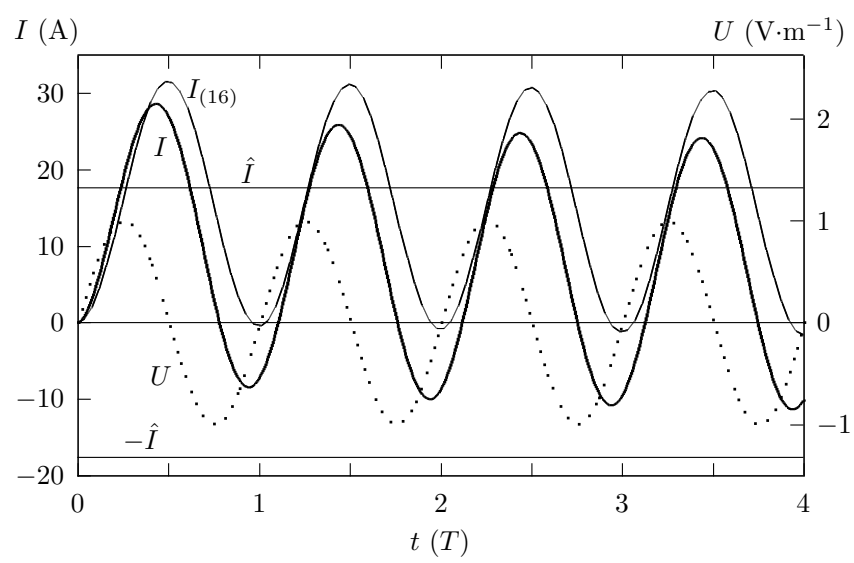

Figure 9. Dependence of the source voltage $U$ and transient currents $I_{(16)}$ and $I$ on $t \in[0,4 T]$. The symbol $\hat{I}$ indicates the steady current amplitude $(t \rightarrow \infty)$.

\section{Conclusions}

Two long parallel conductors with the voltage source form a long loop. A method for calculating current density in a group of long parallel conductors has recently been published [1]. This method was derived rigorously from knowledge whose validity cannot be doubted. The derivation of the method is based, in the first place, on Faraday's law of electromagnetic induction and is an application of the Biot and Savart law, loop current method, Kirchhoff's voltage law, Ohm's law, the Jordan measure theory and graph theory. The essence of the method consists in the conductors being replaced by partial conductors of rectangular cross section and constant current density. The partial conductors form partial loops. One metre of each partial loop is replaced by a series resistor-inductor circuit. The interaction of partial loops through magnetic field is considered. To calculate current density in the partial conductors, it is not necessary to specify the inductances of inductors in the partial loops. The transient current in the loop is at each instant equal to the flux of transient current densities in all the partial loops.

The method described in [1] is extended to a form suitable for numerical calculation of transient current density. The possibilities of the proposed method are demonstrated by solving illustrative examples. The method is used for an accurate calculation of transient current using transient current density. The transient current is analysed when connecting and short-circuiting the sources of sinusoidal, constant and sawtooth voltages. For circular cross section conductors, the dependences of maximum current density, maximum current and the time of achieving steady state on the source frequency, the distance of the conductors and their resistivity when connecting the source of sinusoidal voltage are examined.

Funding: This research work was carried out in the Centre for Research and Utilization of Renewable Energy (CVVOZE). The author gratefully acknowledges financial support from the Ministry of Education, Youth and Sports of the Czech Republic under Brno University of Technology specific research programme (project No. FEKT-S-20-6449).

Conflicts of Interest: The author declares no conflict of interest. The funder had no role in the design of the study; in the collection, analyses, or interpretation of data; in the writing of the manuscript, or in the decision to publish the results.

\section{References}

1. Coufal, O. Current density in a group of long parallel conductors. Phys. Scr. 2019, 94, 125504. [CrossRef]

2. Coufal, O.; Radil, L. Self-induction in two long parallel conductors connected to sinusoidal voltage source. Phys. Scr. 2020, 95, 065503. [CrossRef]

3. Coufal, O. Faraday's law of electromagnetic induction in two parallel conductors. Int. J. Appl. Electromagn. Mech. 2017, 54, 263-280. [CrossRef] 
4. Riba, J.R. Analysis of formulas to calculate the AC resistance of different conductors' configurations. Electr. Power Syst. Res. 2015, 127, 93-100. [CrossRef]

5. Freitasc, D.; Guerreiro das Nevesa, M.; Almeidab, M.E.; Maló Machadoa, V. Evaluation of the longitudinal parameters of an overhead transmission line with non-homogeneous cross section. Electr. Power Syst. Res. 2015, 119, 478-484. [CrossRef]

6. Shafieipoura, M.; Chenb, Z.; Menshovc, A.; De Silvaa, J.; Okhmatovskib, V. Efficiently computing the electrical parameters of cables with arbitrary cross-sections using the method-of-moments. Electr. Power Syst. Res. 2018, 162, 37-49. [CrossRef]

7. Mohos, A.; Ladányi, J.; Divényi, D. Methods to ascertain the resistance of stranded conductors in the frequency range of $40 \mathrm{~Hz}-150 \mathrm{kHz}$. Electr. Power Syst. Res. 2019, 174, 105862. [CrossRef]

8. Rahman, M.S. Basic Graph Theory eBook; Springer International Publishing AG: Berlin/Heidelberg, Germany, 2017. [CrossRef]

9. Coufal, O.; Bátora, B.; Radil, L.; Toman, P. Simple calculation of eddy currents in a long passive conductor. Int. J. Appl. Electromagn. Mech. 2019, 61, 201-224. [CrossRef]

10. Rektorys, K.; Vitásek, E. Survey of Applicable Mathematics; Kluwer Academic Publishers: Dordrecht, The Netherlands, 1994.

11. Ralston, A.; Rabinowitz, P. A First Course in Numerical Analysis; Dover Publications, Inc.: Mineola, NY, USA, 2001.

12. Kamke, E. Differentialgleichungen, Lösungsmethoden und Lösungen; Vieweg + Teubner Verlag: Wiesbaden, Germany, 1977.

13. Lide, D.R. (Ed.) CRC Handbook of Chemistry and Physics, 88th ed.; CRC Press: Boca Raton, MA, USA, 2007.

14. Shenkman, A.L. Circuit Analysis for Power Engineering Handbook; Kluwer Academic Publishers: Dordrecht, The Netherlands, 1998.

15. Maxwell, J.C. A Treatise on Electricity and Magnetism, 1st ed.; Clarendon Press: Oxford, UK, 1873; Volume II.

16. Dlabač, T.; Filipovič, D. Integral equation approach for proximity effect in a two-wire line with round conductors. Tech. Gaz. 2015, 22, 1065-1068. [CrossRef]

17. Raven, M.S. Experimental measurements of the skin effectand internal inductance at low frequencies. Acta Tech. 2015, 60, 51-69.

18. Riba, J.R. Calculation of the ac to dc resistance ratio of conductive nonmagnetic straight conductors by applying FEM simulations. Eur. J. Phys. 2015, 36, 055019. [CrossRef]

19. Kim, J.; Park, Y.J. Approximate Closed-Form Equation for Calculating Ohmic Resistance in Coils of Parallel Round Wires With Unequal Pitches. IEEE Trans. Ind. Electron. 2015, 62, 3482-3489.

20. Riba, J.R.; Capelli, F. Calculation of the inductance of conductive nonmagnetic conductors by means of finite element method simulations. Int. J. Electr. Educ. 2020, 57, 230-252. [CrossRef]

21. Aebischer, H.A.; Friedli, H. Analytical Approximation for the Inductance of Circularly Cylindrical Two-Wire Transmission Lines with Proximity Effect. Adv. Electromagn. 2018, 7, 25-34. [CrossRef]

22. Jabłoński, P.; Szczegielniak, T.; Kusiak, D.; Piątek, Z. Analytical-Numerical Solution for the Skin and Proximity Effects in Two Parallel Round Conductors. Energies 2019, 12, 3584. [CrossRef]

23. Jabłoński, P.; Kusiak, D.; Szczegielniak, T.; Piątek, Z. The Proximity Effect in Twin Line with Round Conductors Placed in Conductive Medium. Energies 2020, 13, 6087. [CrossRef]

24. Coufal, O.; Radil, L.; Toman, P. Magnetic field and forces in a pair of parallel conductors. Int. J. Appl. Electromagn. Mech. 2018, 56, 243-261. [CrossRef] 